\title{
During or After
}

National Cancer Institute

\section{Source}

National Cancer Institute. During or After. NCI Thesaurus. Code C49640.

Within a certain period of time or after a certain point or period in time. 